http://doi.org/10.15359/ree.12-1.9

\title{
UNA REFLEXIÓN NECESARIA: POSIBILIDAD DE LA CONSTRUCCIÓN DE UN MODELO PEDAGÓGICO EN LA EDUCACIÓN SUPERIOR
}

\author{
Ileana Castillo Cedeño ${ }^{l}$ \\ Vicedecana del CIDE, Universidad Nacional \\ Heredia, Costa Rica
}

Luz Emilia Flores Davis ${ }^{2}$

Docente e Investigadora de la División de

Educación Básica del CIDE-Universidad Nacional

Heredia, Costa Rica

\begin{abstract}
Rafael Esteban Jiménez Corrales ${ }^{3}$
Docente e investigador en la División de Educación Básica del CIDE-Universidad Nacional

Heredia, Costa Rica

Mariángeles Perearnau Torras ${ }^{4}$

Docente e investigadora en la División de Educación Básica del CIDE-Universidad Nacional

Heredia, Costa Rica
\end{abstract}

Recibido 15 de mayo 2007 • Aprobado: 19 de junio 2007

\begin{abstract}
Resumen: consideraciones en torno a la pertinencia de establecer un modelo pedagógico en una institución de educación superior universitaria, así como los aspectos que deben caracterizarlo, con el propósito de orientar los procesos educativos hacia nuevas perspectivas críticas. Lo anterior con fundamento en los resultados de una investigación llevada a cabo por un equipo académico de la Universidad Nacional.
\end{abstract}

Palabras clave: Modelo pedagógico, Educación superior universitaria.

Abstract: The current article presents a series of considerations regarding the significance of establishing a pedagogic model in University level education, as well as its main characteristics, this with the purpose of guiding educational processes towards new and critical perspectives. The article is based on the results of an investigation performed by a team of professionals at the Universidad Nacional.

Key words: Pedagogic model, University level education.

\footnotetext{
Investigadora, docente y coordinadora de educación continua en la División de Educación Básica del CIDE de la Universidad Nacional, Heredia, Costa Rica. Doctora en Mediación Pedagógica. Ha laborado en distintos niveles del sistema educativo desde preescolar hasta el universitario, así como también con diversas poblaciones entre las que destacan adolescentes de comunidades en desventaja social y adultos mayores.

2 Docente, investigadora y extensionista en la División de Educación Básica del CIDE y docente en la Maestría en Formación Dancística del CIDEA, Universidad Nacional, Heredia, Costa Rica. Máster en Educación Superior y catedrática universitaria. Ha ocupado los cargos de Directora de Docencia de la Universidad Nacional y Directora de Desarrollo Profesional y Cooperación Universitaria en esa misma institución. Entre sus publicaciones más recientes destacan trabajos para la Alfabetización de personas adultas.

3 Docente, investigador en la División de Educación Básica del CIDE, Universidad Nacional, Heredia, Costa Rica. Máster en Evaluación Educativa y Licenciado en Historia. Ha ocupado cargos de Subdirector de la División de Educación Básica del CIDE y Coordinador de la Maestría en Pedagogía con énfasis en Diversidad en los procesos Educativos. Además formó parte del equipo de Diseño Curricular de la Dirección de Docencia en esa misma institución.

4 Docente e investigadora en la División de Educación Básica del CIDE de la Universidad Nacional, Heredia, Costa Rica. Licenciada en Psicología. Ha ocupado distintos cargos en el Instituto sobre Alcoholismo y Farmacodependencia entre los que destacan: psicóloga de consulta externa, coordinadora de la Clínica Infanto Juvenil y Jefe de Prevención a nivel Nacional.
} 
El trabajo cotidiano de la enseñanza y el aprendizaje, en los diferentes niveles del sistema educativo, genera conocimiento que, si bien enriquece a las personas que participan de la experiencia pedagógica, no siempre se traduce en el desarrollo de la pedagogía. De ahí la importancia de la investigación educativa y de la divulgación de sus resultados.

La intencionalidad de este artículo consiste en compartir la teorización llevada a cabo por un equipo de académico, docentes e investigadores, a partir de un proceso de investigación realizado en el marco de un proyecto de la División de Educación Básica, del Centro de Investigación y Docencia en Educación, CIDE, de la Universidad Nacional en Costa Rica, cuyo constructo fundamental lo constituía el modelo pedagógico.

Es de justicia académica reconocer los aportes, en la construcción de este artículo, de la máster Ana Lorena Vargas Víquez, quien participó en el desarrollo de la investigación. Muchas de sus ideas se plasman en el documento, pues su ser crítico e innovador permeó al resto del equipo y lo hizo permanente.

\section{CONCEPTUALIZACIÓN DEL CONSTRUCTO MODELO PEDAGÓGICO}

El hecho pedagógico es un acto de creación y, por lo tanto, un acto profundo con un alto nivel de incertidumbre (concetualizada dentro de la lógica de la teoría del caos). No obstante, es posible identificar una serie de rasgos que caracterizan maneras variadas de abordar la acción pedagógica, lo cual permite distinguir diversos modelos.

Existen diferentes definiciones de la palabra modelo. Para los efectos de este trabajo, el concepto de modelo se entiende con Flórez (2000), como representación de las relaciones que describen un hecho. El modelo constituye una "imagen" que permite profundizar en la comprensión de lo que representa. Interesa el concepto de modelo en tanto sirve como "espejo" para identificar la propia práctica pedagógica. Sin embargo, no debe buscarse una correspondencia absoluta entre las características de un modelo pedagógico determinado, y la forma en cómo se aborda la acción en el aula; sino más bien, el modelo puede servir para analizar la acción educativa a la luz de ciertas características generales correspondientes a una visión pedagógica particular, y constituirse, entonces, en faro orientador de la práctica.

Un modelo se organiza a manera de sistema formado por elementos, lo que significa que una modificación en alguno de ellos, tiene un impacto en todos los otros y en la totalidad del modelo. Utilizar un modelo para el análisis de la práctica pedagógica, requiere la capacidad de relacionar todos sus elementos, los cuales dan cuenta tanto de la realidad empírica como de los supuestos epistemológicos que la fundamentan.

Sin embargo, ante la dificultad de realizar generalizaciones de realidades tan complejas como los hechos educativos, es indispensable visualizar el modelo pedagógico como estructura dinámica que permite abordar, desde sus supuestos, el análisis de expresiones socio-históricas específicas y concretas, donde se dan las interacciones entre la enseñanza intencional y el aprendizaje. Por lo anterior, no puede pretenderse que de un modelo pedagógico se deriven estrategias de enseñanza que se apliquen de manera mecánica, sino espacios para un trabajo docente reflexivo, en el marco de principios orientadores. Pensar de otra forma es colocarse en una posición tradicional caracterizada por la mutilación de la acción propositiva de las y los docentes.

Reflexionar sobre la práctica docente constituye un paso importante en su mejoramiento; pero sólo cuando se está abierto a escuchar diversas voces, a incorporar en el análisis diferentes 
perspectivas y a cuestionar lo que se da por cierto, se está en condiciones de superar las prácticas rutinarias que no corresponden a los ideales, y se inicia la construcción de los cimientos para una reconceptualización del diálogo.

\section{CLASIFICACIÓN DE LOS MODELOS PEDAGÓGICOS}

Tomando en cuenta la perspectiva que se ha expuesto respecto a la conceptualización adoptada sobre el término modelo y a las limitaciones que todo instrumento de análisis presenta para dar cuenta de realidades complejas formadas por prácticas únicas, se pueden mencionar los siguientes modelos pedagógicos:

- Modelo pedagógico tradicional

- Modelo pedagógico conductista

- Modelo pedagógico romántico

- Modelo pedagógico cognitivo

- Modelo pedagógico social

Antes de presentar cada uno de los modelos enunciados, se quiere reiterar que el modelo, como faro orientador, siempre será teórico, pero para el abordaje de la práctica pedagógica no existe un modelaje, no hay un único camino, sino que cada persona y en cada experiencia, debe desplegar un estilo o estilos propios.

\section{Modelo pedagógico tradicional}

Un aspecto fundamental del modelo pedagógico tradicional es la autoridad casi imperiosa que se le atribuye al libro y al educador o educadora. Desde el punto de vista del ser humano que se quiere formar, este modelo enfatiza en la adquisición de valores humanistas o religiosos, asociados con el carácter de enseñanza absoluta, más que a la búsqueda de la verdad en un espíritu de libertad. Se recalca la transmisión de saberes del educador, que los transfiere al estudiante como verdades acabadas, y que en ningún caso, pueden ser cuestionadas por este. Los conocimientos son atemporales y la relación docente-estudiante se da en una sola vía; no se promueven las relaciones entre estudiantes en los procesos educativos formales. Las diferencias individuales y las experiencias personales de quienes intervienen en el proceso educativo no son consideradas aspectos esenciales.

\section{Modelo pedagógico conductista}

En el modelo conductista se transmiten los saberes que son aceptados socialmente. De acuerdo con este modelo, la meta de un proceso educativo es el moldeamiento de las conductas que se consideran adecuadas y técnicamente productivas, según los parámetros sociales establecidos.

La y el docente presentan situaciones de aprendizaje en las que los estímulos se programan para lograr las respuestas deseadas. Los objetivos deben estar establecidos de forma clara y se enseña para obtener su logro. Al ser una enseñanza "de fuera hacia adentro", se puede aprender por 
un sistema organizado de prácticas o repeticiones reforzadas adecuadamente. El aprendizaje puede tener un carácter activo, manipulando elementos del medio, para lograr una conducta programada.

Se fomenta la enseñanza individualizada y el trabajo en pequeños grupos. En épocas modernas, se introduce el requerimiento de que el ambiente esté dotado de diversos recursos tecnológicos para ser utilizados en el acto educativo.

\section{Modelo pedagógico romántico}

El modelo pedagógico romántico puede situarse, por sus principios, en el extremo contrario al modelo pedagógico conductista. De esa forma, en dicho modelo se habla de libertad, de autonomía, de creatividad.

Se busca el desarrollo natural de la persona, su expresión sin inhibiciones impuestas por el medio. Por lo tanto, el papel del o la docente es de auxiliar o apoyo a la libre expresión de sus estudiantes. Son estos quienes llevan el liderazgo pedagógico de acuerdo con sus intereses.

En el modelo pedagógico romántico no tienen sentido la programación ni la evaluación, sino el desarrollo de los procesos que genera las decisiones que se van tomando. A lo más que se podría llegar sería a procesos de observación no participante, como formas de registro del desarrollo educativo natural del ser humano.

\section{Modelo pedagógico cognitivo}

En cuanto al ser humano que se quiere formar, el modelo pedagógico cognitivo es individualista; enfatiza el logro de conocimientos de parte de la persona. Se acentúa el conocimiento y se considera que cada sujeto puede conocer, de manera diferente, la realidad. El maestro debe orientar a su estudiante a desarrollar aprendizajes significativos a participar en actividades exploratorias que puedan ser usadas posteriormente en formas de pensar independiente. Toma relevancia la experiencia del estudiante, lo que implica conocer y aceptar sus vivencias para lograr la construcción de nuevos conocimientos que sean relevantes.

Se promueve la realización de experiencias prácticas y de experimentación, por lo que la enseñanza se lleva a cabo con una cuidadosa planificación y selección de actividades, en la que resulta de gran importancia que las y los estudiantes se involucren y comprometan. Por otra parte, el modelo cognitivo apuesta por un ambiente estimulante de experiencias que faciliten el desarrollo de estructuras cognoscitivas. Se otorga libertad y oportunidades para que se interactúe con el objeto de estudio. Se suscita una participación activa y constructiva a partir de la información que proporciona el medio.

\section{Modelo pedagógico social}

En el modelo pedagógico social, se busca posibilitar el incremento de la libertad y el logro de cambios en la sociedad. Interesa el carácter político de la educación. Se favorece el crecimiento personal de las y los estudiantes en su contexto familiar, escolar y comunitario, además, interesa la crítica a las estructuras que afectan la vida escolar. En este modelo, se propicia el desarrollo de 
habilidades de pensamiento crítico-reflexivo que permitan el cambio social. Pero no es solamente un lenguaje de crítica, sino de posibilidades. Las y los docentes coparticipan con sus estudiantes en la reflexión crítica de sus propias creencias y juicios, así como en el análisis de su realidad.

Se impulsa el trabajo en grupos para la atención de problemas correspondientes con la realidad concreta de los estudiantes, en su contexto social. Por todo lo expuesto, en el modelo pedagógico social se precisa un educador capaz de cuestionarse, abrirse al cambio y vivenciar coherentemente su discurso. El intercambio se realiza en un marco de tolerancia, diálogo real y respeto a las ideas de los demás; así, las ideas divergentes, con respecto a posiciones tradicionales y conductistas, encuentran un espacio para su desarrollo en este modelo pedagógico.

Cabe destacar que desde el punto de vista conceptual, es posible identificar elementos que sugieren complementariedad entre el modelo pedagógico cognitivo y el social, a pesar de sus diferencias en cuanto al propósito educativo central de cada uno.

\section{UN MODELO PEDAGÓGICO EN EL CONTEXTO UNIVERSITARIO}

¿Es conveniente que una institución universitaria opte por un modelo pedagógico? Y de ser así, ¿es ello posible?

La universidad contemporánea, por definición, alude a lo universal, a la búsqueda de la totalidad, trasciende lo local, la específico, con la pretensión de abrir horizontes, de generar y compartir conocimientos, de formar personas capaces de incidir en la conformación de un determinado tipo de sociedad. Sin embargo, hay que tener presente que la universidad puede optar por constituirse en gendarme de la organización social dominante o en agente de cambio. Por tratarse de una institución educativa, la universidad nunca será ideológicamente neutra.

Por su autonomía, las universidades estatales tienen la posibilidad de constituirse en espacios para el libre debate sobre los grandes problemas sociales. Tienen el reto de buscar, junto con otras organizaciones, la construcción de una sociedad más justa y equitativa. Desde el punto de vista de este artículo, las universidades públicas tienen la responsabilidad de generar procesos que coadyuven al desarrollo humano con equidad. En este sentido, cobra relevancia la definición de un modelo pedagógico que concrete una determinada visión educativa, un modelo que oriente el quehacer universitario hacia los grandes fines y funciones, así como al cumplimiento de su misión.

La universidad legitima su modelo pedagógico en la medida que dé cuenta en su accionar, de los propósitos que le dan sentido y razón de existir. En consideración a lo anterior, cabe señalar que no basta con la definición o adopción teórica de un modelo pedagógico determinado, sino que es imprescindible que éste resulte de la participación y compromiso de la comunidad universitaria.

El modelo pedagógico, como representación de las relaciones que describen un hecho, constituye una imagen que permite profundizar en la comprensión de lo que simboliza, y como la universidad es una institución dinámica, en la cual se llevan a cabo procesos educativos complejos, resulta indispensable que se asuma el modelo pedagógico como estructura flexible que permita abordar el análisis de expresiones socio-históricas en constante actualización.

No puede pretenderse que de un modelo pedagógico se deriven estrategias de enseñanza que se apliquen de manera mecánica, sino más bien, espacios para un trabajo docente reflexivo en el marco de principios orientadores. Para que la reflexión genere posibilidades de cambio, esta debe tomar en cuenta las distintas perspectivas de todas las personas que participan en el hecho educativo, lo que requiere aceptar y respetar puntos de vista divergentes. 
Lo anterior necesita de la realización de procesos permanentes de desarrollo profesional de las y los funcionarias universitarios. La actualización en la disciplina propia de cada académica y académico, la formación pedagógica y el crecimiento integral de cada persona, a partir de la visión y misión definidas por la instancia, se integran para garantizar docentes universitarios que respondan a las expectativas que la sociedad le confiere. Por otra parte, no puede olvidarse la importancia de la integración de las áreas académicas: docencia, investigación, extensión y producción, lo que plantea una dimensión distintiva de las y los profesionales que laboran en una institución de educación superior universitaria.

La legitimación de la educación universitaria se construye en la medida en que se demuestra la calidad de sus procesos y resultados. Indudablemente, entran en juego múltiples condicionantes relacionadas con la teoría y práctica educacional, entre las que destacan: la ética profesional de todas las personas involucradas en el proceso educativo, la disposición de recursos para la investigación y la innovación, el conocimiento de necesidades y condiciones de la población a la que sirven, la pertinencia cultural del currículo, la claridad en su gestión y el alcance de su mirada. Todas estas variables apuntan al complejo ámbito de relaciones humanas, a los significados culturales y sociales que se edifican en la cotidianidad de la práctica universitaria.

Comprender el sentido de la práctica pedagógica se convierte en un elemento vital para redimensionar el papel formativo y ético que tiene la educación superior en tiempos de incertidumbre y de crisis. Implica habilitar un espacio abierto al debate y al diálogo en torno a la calidad y equidad de la enseñanza. Supone dar paso a la deliberación y al escrutinio crítico que deben sustentar todo proyecto educativo, para ampliar el significado construido hasta hoy desde prácticas diversas, con el afán de perfilar alternativas educativas que contribuyan a las transformaciones profundas que requiere nuestra educación y nuestra sociedad.

Conlleva también, el análisis exhaustivo, minucioso, transversal, de la pluridimensionalidad de factores implícitos y explícitos en los diversos ámbitos de la planificación y organización universitaria, teniendo presente que estos se convierten en elementos decisorios de modelos de pensar y actuar.

Las universidades, en general, tienen la necesidad y responsabilidad de repensar los procesos que desde ellas se gestan en función de configurar contextos educacionales diversificados. Para ello, se requiere de un pensamiento inclusivo, capaz de interpretar la realidad compleja e intervenir en esta, de manera consciente.

En primera instancia, se deberían considerar, o bien repasar, aquellos elementos que tentativamente constituyen un modelo pedagógico:

1. Tipo de sociedad y ser humano que se pretende generar.

2. Conceptuación de los procesos de aprendizaje.

3. Tipos de relaciones generadas en el proceso educativo.

4. Tipo de estrategias mediadoras en el proceso de enseñanza.

5. Papel de los contenidos temáticos.

6. Función y concepción de la evaluación.

El primer punto es fundamental de considerar, ya que tiene que ver directamente con el porqué del desarrollo un proceso educativo e implica cuestiones onto-axiológicas. Lo anterior se relaciona con el pasado, con la historicidad de una institución, así como su futuro.

Si nos referimos a una institución educativa, se debe mirar hacia atrás las hojas del calendario y repensar para qué se creó, ¿cuál fue su origen?, o en términos más empresariales ¿cuál fue 
su misión inicial? No obstante, una vez que se responda a esas preguntas, cabría cuestionarse si la intencionalidad originaria es la misma que se desea en el presente y si es posible entrever una mirada hacia el futuro, y pensar si será la intencionalidad del futuro. Es decir, se ha de pensar que las instituciones no son invariantes, sino dinámicas. No obstante, es necesario analizar si esa dinamicidad es tal como la misión.

\section{EL CASO DE LA UNIVERSIDAD NACIONAL DE COSTA RICA}

La Universidad Nacional nace (dejando de lado otros entretelones políticos) con la claridad meridiana de dinamizar a los sectores desposeídos de Costa Rica. Con el lema de la "Universidad Necesaria"; se busca absorber y potencializar aquellos sectores sociales que a través de la educación no habían podido movilizarse, verticalmente, en la Costa Rica cuyo modelo de desarrollo se caracterizaba por la presencia de un estado interventor.

Surgen, entonces, las preguntas: ¿desaparecieron esos sectores?, ¿se dinamizó de tal forma el modelo socioeconómico que hizo que esos sectores se catapultaran a mejores condiciones de vida?, ¿es que ya no existen sectores de atención prioritaria que requieran del apoyo y soporte de una universidad necesaria?

La respuesta es contundente: los sectores desposeídos crecieron en la Costa Rica del siglo XXI. Dichos sectores se han multiplicado y visibilizado. No sólo es la Costa Rica de pequeños productores ahogados por falta de ayuda y soporte técnico, sino que es la Costa Rica de mujeres agredidas, de irrespeto a las diferencias, de seres humanos adiestrados para consumir, de personas frustradas por no poder consumir, de violentas muertes, de desconfianza de la clase política, de inmigrantes con muy bajas oportunidades de desarrollo, ascenso socioeconómico y de respeto a sus diferentes identidades. En conclusión, los principios a los que respondía la Universidad Nacional son ahora más pertinentes, más visibilizados y necesarios.

Conocidos los diferentes modelos pedagógicos, es obvio que la situación mundial, latinoamericana y nacional, llevarían a elegir principios pedagógicos que respondan a la situación dada en estos momentos. Principios que no pueden alejarse de los originarios, principios que tengan relación directa con una Universidad Necesaria, pero necesaria para los sectores excluidos del país y de Centroamérica.

Cuando se piensa en un modelo pedagógico emparentado con el concepto de una Universidad Necesaria, conduce directamente a imaginar un modelo que al menos cumpla con los siguientes principios:

1. Un modelo que busque promover el respeto hacia los otros, que tenga como ideal la igualdad de oportunidades y la contribución a una sociedad más justa y equitativa, en la cual, tanto hombres como mujeres, vivan los principios de solidaridad, ayuda mutua y cooperación. Una sociedad en donde se pueda discrepar, donde la comunicación y la información jueguen papeles decisivos, donde los seres humanos puedan vivir en paz con el planeta y consigo mismos.

2. Para la promoción de tal sociedad y sus seres humanos, se requiere conceptuar el aprendizaje como proceso sociocultural, histórico, mutable, posible, lúdico, con un sentimiento de gozo creativo. Debe partirse de la multiplicidad de formas de aprender, por lo tanto, de la multiplicidad de formas de enseñar. 
3. La promoción de tal sociedad requiere de principios que fomenten procesos participativos, aulas participativas donde las verdades sean discutidas por los actores y por las actrices involucrados, donde las argumentaciones y los procesos conjuntos de construcción y clarificación sean guías de los procesos de aula e institucionales.

4. Lo anterior requiere variedad en los procesos de mediación pedagógica, la utilización de diversos medios y estrategias de enseñanza, del respeto a las diferentes capacidades y discapacidades. Es necesaria la formación docente permanente en procesos didácticos que no atenten contra la misión original de la universidad.

5. Los contenidos temáticos atinentes para el tipo de modelo que se ha venido perfilando, no pueden ser considerados como verdades absolutas. Siguiendo los procesos de construcción de cada una de las diferentes ciencias, se requiere comprender sus principios lógicos subyacentes. Con ello, se persigue el desarrollo de capacidades que permitan la superación de una formación materiocéntrica, y que lleven a la construcción de estructuras de pensamiento y a la posibilidad de mirar los cambios en su disciplina y proponer soluciones ante ellos. Esto implica un componente fuerte en capacidades de análisis sociocultural e histórico, visiones instrumentalistas quedan fuera del modelo pedagógico necesario.

6. Todo lo anterior se debe plasmar en los procesos evaluativos, los cuales como parte fundamental del currículum, no pueden verse como accesorios a este. Por tal razón, la evaluación debe mirar los procesos y productos, orientarse hacia enfoques cualitativos y quizá hacer la ruptura con las prácticas de medición tradicionales. Esa evaluación requiere ser formación misma, demanda crear la cultura de autoevaluarse y coevaluarse. Además, pretende ser una evaluación que desborde los procesos de enseñanza y aprendizaje; se necesita una evaluación participativa de la gestión, que en términos generales, se debe fundamente en el principio de reflexión. Y como lo señala Freire, debe existir la "humildad académica", que señala que se está en proceso constante de aprendizaje, que pide poder mirar en el espejo de la crítica y someterse a ella; una evaluación que trascienda la justificación y que recuerde que la acción de la enseñanza es más un arte, y por lo tanto, perfectible en forma constante.

Comentados los aspectos anteriores, la conclusión es obvia: son los modelos sociales-cognitivos los que permiten el desarrollo de una institución universitaria que tenga como destino histórico ser una Diversidad Necesaria y pertinente para la inclusión y promoción de los sectores menos favorecidos del país y del área. Tales modelos contemplan la posibilidad de discusión y de acuerdos, ahí nace quizá su mayor nivel de pertinencia. No obstante, el asumir tal modelo implica cambios institucionales estructurales, y sobre todo, una amplia reflexión pedagógica de parte de todos los sectores involucrados en la vida universitaria.

\section{EDUCACIÓN Y CURRÍCULO: SU SIGNIFICADO Y CONSTRUCCIÓN EN EL CON- TEXTO DE UN MODELO PEDAGÓGICO UNIVERSITARIO}

A la pregunta eje: ¿es posible la creación de un modelo pedagógico para una institución de educación superior universitaria?, se plantea, tal como se ha descrito anteriormente, un modelo pedagógico amplio, flexible, crítico, que desde una dimensión pluralista de la diversidad permita a la universidad realizar un serio esfuerzo educativo en el sentido que bien señala Olivé, de enseñar 
de nuevo a pensar críticamente y promover la reflexión sobre los valores y los objetivos que orientan los actuales sistemas educativos.

En cuanto al currículo que facilite caminar en la construcción de dicho modelo pedagógico, probablemente no haya un solo diseño, sino diseños que se orienten hacia el logro común de una sociedad desarrollada con fundamento en la educación de toda su ciudadanía, bien sea mediante acciones formales, informales, educación escolarizada, docencia, extensión o investigación universitaria, entre otras.

Kemmis (1986) señala que en las sociedades existen actualmente grandes discrepancias en la comprensión del significado y funciones del currículo, sobre todo en la búsqueda de una práctica educativa más reflexiva y comprometida con el logro de una sociedad más justa y equitativa. En este sentido, el currículo asume el papel de gestor y facilitador de la diversidad y de la equidad.

Los principios que se sugieren para sustentar un modelo pedagógico universitario son válidos para el diseño curricular de las carreras universitarias, sobre todo aquellos que consideren, como bien señala Gimeno(2001), la necesidad de restablecer ese sentido esencial de la educación y su compromiso con ésta.

Una opción de currículo para una institución de educación superior universitaria, podría ser diseñado como una hipótesis perfectible, como una construcción social, histórica y cultural, para que permita, en el caso de la Universidad Nacional, recuperar su razón de ser en el devenir de la historia costarricense; que su desarrollo permita la formación de profesionales comprometidos a su vez con la diversidad y la equidad en el espacio profesional en que se desenvuelven.

Para una institución universitaria es importante clarificar sus políticas en el campo de la planificación curricular. Indiscutiblemente, la naturaleza de las diversas disciplinas que se desarrollan no puede ser sometida a criterios de homogeneidad mediante directrices para el diseño del currículo, porque el quehacer universitario puede ser neutralizado mediante tecnicismos del currículo que lo convierten en un fin en sí mismo.

El currículo es un medio y, como bien dice Stenhouse (1988), hay que descartarlo como un producto pensado burocráticamente, y hoy, las instituciones universitarias, en aras de un supuesto control de calidad, tienden a controlar la academia vía diseño del currículo. En este sentido, es importante citar a Grundy (1987), quien afirma que no es corriente que se parta de cero en cuestiones curriculares, es decir, que "...tanto profesores como alumnos están ya comprometidos en práctica curriculares” (p. 20). Pensar en currículo, continúa Grundy (1987), es pensar cómo actúan e interactúan las personas en determinadas situaciones.

La propuesta de diseño problematizador de Magendzo, parece un diseño curricular viable para un modelo universitario, porque requiere que el profesorado, al apropiarse de ésta, asuma una acción docente crítica y autónoma, principios básicos para la docencia universitaria.

En todo caso, si el currículo se interpreta como una construcción social, histórica y cultural, el profesorado y alumnado deberán ser protagonistas de su diseño y no simples ejecutores de decisiones burocráticas que involucran, no obstante, visiones de ser y estar en el mundo, las cuales, inconscientemente, legitiman una comunidad universitaria, cuando ha sido construido desde una perspectiva técnica, como producto de departamentos administrativos universitarios.

Para concluir estas consideraciones sobre la pertinencia de un diseño curricular para un modelo pedagógico universitario, es importante rescatar que lo relevante es la claridad de una visión educativa para los tiempos actuales en un contexto de justicia y equidad. Con ello, permitir la acción de toda la comunidad universitaria desde la consideración de la diversidad como derecho y valor para la convivencia democrática en una sociedad. 
Sin embargo, es necesario señalar aquellos diseños y prácticas curriculares que no corresponden a ningún nivel educativo, menos al universitario; nos referimos a los enfoques asimilacionistas del currículo, validados luego de su aplicación por procesos de medición que consolidan posiciones conservadoras de obediencia debida en el profesorado y alumnado universitario; es decir, que utilizan el currículo como una teoría y una práctica de y para la reproducción.

\section{UN CURRÍCULO UNIVERSITARIO PERTINENTE}

Se requiere de mecanismos de descentralización curricular para dinamizar el proceso, de forma tal que toda la comunidad universitaria participe de manera activa en el diseño, implementación y evaluación. Cabe recordar que el currículo es entendido como construcción humana que responde a una realidad multidimensional. Por ello, se convierte en un proceso de negociación y transformación permanente.

Como se mencionó con anterioridad, debe ser coherente con las demandas de una sociedad emergente que reclama perfilar nuevos campos de formación humana para posibilitar su movilidad social ascendente y favorecer el rompimiento de ciclos de marginación económica, social, cultural y política.

Un currículo universitario pertinente, entonces, tendrá que responder a la diversidad de personas y contextos. Su estructura y los mecanismos de funcionamiento deben potencializar la participación y el compromiso real centrados en el diálogo, en la investigación interdisciplinar y transdisciplinar, que permitan niveles de análisis más profundos y alternativas educativas innovadoras, que posibiliten una apropiación efectiva de la cultura y de la vida.

Lo anterior ha de ser evidente en los planes y programas de estudio, que tienen que constituirse en un proceso y un producto democrático al servicio del ser humano. Estos deben ser explícitamente vinculantes con la vida, para lo cual se requiere la creación de redes de comunicación y apoyo entre unidades académicas, facultades y otras instancias gubernamentales y no gubernamentales, que permitan mantener vigente el debate académico en torno a la realidad circundante.

No cabe la menor duda de que la organización de prácticas de enseñanza, en cualquier nivel del sistema educativo, sugiere una honda transformación en los estilos habituales de aprender y enseñar. Desde esta perspectiva, la formación del profesorado universitario debería acentuarse en la reflexión sobre su propia acción, para favorecer una enseñanza basada en la comprensión y la ética.

Asumir una identidad pedagógica crítica, se basa en la autorreflexión, autovaloración, en la crítica constante del quehacer cotidiano, en el intercambio comunicativo que favorezca una liberación en cuanto a limitaciones ideológicas, que inexorablemente operan en el acto educativo.

En este sentido, el y la profesional comprometidos con la educación, tendrán como función reflexionar con respecto a los procesos de enseñanza y aprendizaje de sus estudiantes, descentralizando su pensamiento de forma que deje el poder y dé paso a la toma de decisiones compartidas en cuanto a contenidos, metodología, recursos, espacios y tiempos. Es necesario que considere que no todas las personas acceden de la misma forma a la información, y que todos construyen su saber, desde su autonomía.

El profesorado requiere dotarse de competencias comunicativas que den paso a un nuevo orden en desarrollo del pensamiento y la convivencia diaria, donde la experiencia y el conocimiento previo, así como los intereses y las necesidades de los actores del proceso educativo, entren dinámicamente en la danza de construir nuevos referentes teóricos, epistemológicos y experienciales. 


\section{CONSIDERACIÓN DE LOS ESTILOS DE APRENDIZAJE}

¿Qué sentido adquiere plantearse la importancia que tienen los estilos de aprendizaje, cuando se habla de la elección de un modelo pedagógico en un centro de enseñanza superior?

Los estilos de aprendizaje no tienen relación directa con el modelo pedagógico por el que opte la institución; pero según la opción, variará el uso que se haga de ellos, ya que la reflexión crítica hará que se utilicen estos conocimientos para los fines en los que se crea que se debe orientar la enseñanza superior.

Para el profesorado, puede ser útil el autoconocimiento de sus estilos de aprendizaje. En primer lugar, para conocer cómo cada persona accede al conocimiento y cómo, probablemente, lo transmite.

En segundo lugar, les puede interesar conocer cómo las y los estudiantes captan lo que desde la perspectiva docente se considera esencial. Así, si lo creen conveniente, pueden ampliar los recursos didácticos con el fin de comunicarse con un mayor número de estudiantes. A veces, con pequeñas variaciones didácticas que tomen en cuenta los estilos de aprendizaje, se pueden lograr grandes avances.

Sin embargo, también es de utilidad el conocimiento de los estilos de aprendizaje, aunque el profesorado no considere, en ningún momento, variar su propio estilo de enseñanza. Quizá sea suficiente explicitarle al estudiantado que se encuentra en un centro de enseñanza superior, que pueden y deben superar escollos de pensamiento, que deben comparar diversos estilos y extraerconclusiones.

En muchos de las y los estudiantes que acceden a la universidad, todavía predomina el pensamiento operacional concreto y, fruto de la enseñanza superior, se supone que pueden lograr el pensamiento formal o abstracto, a fin de obtener un pensamiento propio, crítico y que se sustente en conocimientos y convicciones.

Para el grupo de docentes de una carrera, conocer y profundizar el tema de los estilos de aprendizaje, forma parte de su desarrollo profesional, ya que le permitirá profundizar en ciertos aspectos de los procesos de enseñanza y aprendizaje que sin esa vertiente, no podrían ser abordados. También para las y los estudiantes, es importante conocer su propio estilo de aprendizaje. En primer lugar, para tener un amplio panorama de la enseñanza universitaria donde se supone se pondrá en contacto con diversas posiciones respecto al conocimiento. Cada docente puede tener su opción y trasmitirla de determinada manera. Para saber qué acepta o rechaza del abanico de posibilidades que se le ofrecen, debe identificar los rasgos fundamentales de cada opción y, para ello, separar lo que comparte vivencial, sensitiva o conceptualmente. En este punto, puede ser de gran ayuda el conocimiento de los estilos de aprendizaje.

Por otra parte, al alumnado le puede ser muy útil el conocimiento de su propio estilo para potenciar el rendimiento de sus horas de estudio, para elegir nuevas estrategias que le posibiliten incorporar más y mejor información, para comprender que el conocimiento que se le ofrece tiene un valor en sí mismo, y que no necesariamente las técnicas o formas de expresarse del profesor o profesora son las que él o ella querría; sin embargo, eso no es obstáculo para su propio crecimiento intelectual. 


\section{LA UNIVERSIDAD ANTE LOS PARADIGMAS ALTERNATIVOS}

\section{Desafíos pedagógicos}

Otra de las grandes cuestiones que se plantean cuando se habla de modelos pedagógicos, se refiere a las nuevas tendencias y paradigmas que generan las economías totalitarias y las nuevas tecnologías de la información y del conocimiento. No se niega que representan retos que deben reflexionar y asumir las universidades como parte de los procesos de generación y transformación del conocimiento y la realidad. No obstante, se sabe que no es la tecnología en sí misma la que genera y transforma la práctica educativa. Algunos analistas, como Castells, plantean que las tecnologías pueden representar un arma de doble filo, en vez de propiciar el cambio, aumentar la rigidez del sistema y perpetuar esquemas tradicionales de enseñanza-aprendizaje.

En este sentido, no sólo se demanda de una actitud de flexibilidad y apertura a las nuevas tendencias de educación virtual y tecnológica, sino también se requiere de una sólida formación para que, verdaderamente, lleguen a incidir en la construcción de modelos pedagógicos renovadores.

Es innegable el papel que corresponde a la universidad de investigar y reflexionar con respecto a los usos y la función y efectos que pueden tener los paradigmas virtuales y tecnológicos en los procesos de enseñanza y aprendizaje. Redefinir su función y las estrategias logra no sólo la interactividad entre el conocimiento y el aprendizaje, sino niveles de proactividad y afectividad entre las personas.

Es indudable que la educación superior tendrá que incorporar, como parte de sus propuestas pedagógicas, las nuevas tendencias tecnológicas y económicas, sino también unirlas con la ética que exige observar la pluralidad de intereses e inquietudes que demandan los estudiantes, las cuales no son puramente de índole instrumental, sino valoracional y actitudinal para encarar, con coraje, su porvenir profesional y su vida personal.

\section{REFERENCIAS}

Flórez, R. (2000). Evaluación Pedagógica y Cognición. Colombia: Mc Graw Hill.

Gimeno, J. (2001). La educación obligatoria: su sentido educativo y social. Madrid: Morata.

Grundy, S. (1994). Producto o praxis del currículum. Madrid: Morata.

Kemmis, S. (1986). El currículum: más allá de la teoría de la reproducción. Madrid: Morata.

Magendzo, A. (1991). Diseño curricular problematizador: Una propuesta para la paz y los derechos humanos. Primera Conferencia de Educación para la Paz. Costa Rica. (Ponencia).

Stenhouse, L. (1988). La Investigación como base de la enseñanza. España: Ediciones Morata. 